\title{
Existence of ground state solutions for quasilinear Schrödinger equations with general Choquard type nonlinearity
}

Yu-bo He ${ }^{1,2^{*}}$, Jue-liang Zhou ${ }^{1}$ and Xiao-yan Lin ${ }^{1,2}$

"Correspondence:

yubmath@163.com

1 School of Mathematics and

Computation Science, Huaihua

University, Huaihua, Hunan, 418000

China

${ }^{2}$ Key Laboratory of Intelligent

Control Technology for

Wuling-Mountain Ecological

Agriculture in Hunan Province,

Huaihua 418000, China

\begin{abstract}
In this paper, we study the following Choquard type quasilinear Schrödinger equation:

$$
-\Delta u+u-\Delta\left(u^{2}\right) u=\left(l_{\alpha} * G(u)\right) g(u), \quad x \in \mathbb{R}^{N},
$$

where $N \geq 3,0<\alpha<N$, and $I_{\alpha}$ is a Riesz potential. By using the minimization method developed by (Tang and Chen in Adv. Nonlinear Anal. 9:413-437, 2020; Willem in Minimax Theorems, 1996), we establish the existence of ground state solutions with general Choquard type nonlinearity. Our results extend the results obtained by (Chen et al. in Appl. Math. Lett. 102:106141, 2020).
\end{abstract}

MSC: $35 J 60 ; 35 J 20$

Keywords: Quasilinear Schrödinger equation; Ground state solutions; Pohozaev identity; General Choquard type nonlinearity

\section{Introduction}

This article is concerned with the following quasilinear Schrödinger equation:

$$
-\Delta u+u-\Delta\left(u^{2}\right) u=\left(I_{\alpha} * G(u)\right) g(u), \quad x \in \mathbb{R}^{N},
$$

where $N \geq 3,0<\alpha<N, I_{\alpha}$ is a Riesz potential (see [16]), and $g: \mathbb{R}^{N} \rightarrow \mathbb{R}$ satisfies

$\left(g_{1}\right) g \in \mathcal{C}(\mathbb{R}, \mathbb{R})$

$\left(g_{2}\right)$ there exists $C>0$ such that

$$
|G(t)| \leq C\left(|t|^{\frac{N+\alpha}{N}}+|t|^{\frac{2(N+\alpha)}{N-2}}\right) ;
$$

(g $\left.g_{3}\right) \lim _{t \rightarrow 0} \frac{G(t)}{|t|^{\frac{N+\alpha}{N}}}=0$ and $\lim _{|t| \rightarrow+\infty} \frac{G(t)}{\left.|t|\right|^{\frac{2(N+\alpha)}{N-2}}}=0$;

$\left(g_{4}\right)$ there exists $s_{0} \in \mathbb{R}$ such that $G\left(s_{0}\right)>\frac{1}{2} s_{0}^{2}$.

(c) The Author(s) 2020. This article is licensed under a Creative Commons Attribution 4.0 International License, which permits use, sharing, adaptation, distribution and reproduction in any medium or format, as long as you give appropriate credit to the original author(s) and the source, provide a link to the Creative Commons licence, and indicate if changes were made. The images or other third party material in this article are included in the article's Creative Commons licence, unless indicated otherwise in a credit line to the material. If material is not included in the article's Creative Commons licence and your intended use is not permitted by statutory regulation or exceeds the permitted use, you will need to obtain permission directly from the copyright holder. To view a copy of this licence, visit http://creativecommons.org/licenses/by/4.0/ 
It is well known that the existence of solitary wave solutions for the following quasilinear Schrödinger equation is a hot problem

$$
i \partial_{t} z=-\Delta z+W(x) z-\psi\left(|z|^{2}\right) z-\Delta l\left(|z|^{2}\right) l^{\prime}\left(|z|^{2}\right) z
$$

where $z: \mathbb{R} \times \mathbb{R}^{N} \rightarrow \mathbb{C}, W: \mathbb{R}^{N} \rightarrow \mathbb{R}$ is a given potential, $l: \mathbb{R} \rightarrow \mathbb{R}$ and $\psi: \mathbb{R}^{N} \times \mathbb{R} \rightarrow \mathbb{R}$ are suitable functions. For various types of $l$ and $\psi$, the quasilinear equation of the form (1.1) has been derived from models of several physical phenomena. For physical background, the readers can refer to $[1,9,11,15]$ and the references therein. If we set the variable $z(t, x)=\exp (-i L t) u(x)$, where $L \in \mathbb{R}$ and $u$ is a real function, then so many papers focused on standing wave solutions for (1.2). The readers can refer to $[5,8,12,13,20]$ and the references therein. As for Choquard type quasilinear Schrödinger equation, there are few papers except for $[3,4,21]$. In [21], a class of quasilinear Choquard equations has been considered via the perturbation method developed by [13], and they showed the existence of positive solution, negative solution, and multiple solutions. Furthermore, the authors [4] established the existence of positive solutions with the periodic potential or bounded potential. In [3], the authors proved the existence of ground state solutions via Jeanjean's monotonic technique [10].

For the following Choquard equation with a local nonlinear perturbation

$$
\left\{\begin{array}{l}
-\Delta u+V(x) u=\left(I_{\alpha} * F(u)\right) f(u)+g(u), \quad x \in \mathbb{R}^{N} \\
u \in H^{1}\left(\mathbb{R}^{N}\right)
\end{array}\right.
$$

under some suitable conditions on $V$, the authors proved the existence of ground state solutions without super-linear conditions near infinity or monotonicity properties on $f$ and $g$ in [6].

To our knowledge, there are no articles to prove the existence of ground state solutions for (1.1) with general Choquard type nonlinearity. In this paper, motivated by [3, 4, 6, 21], we consider the existence of ground state solutions with the Berstycki-Lions conditions, which originated from [2]. To prove our results, we use the minimization method developed by Tang [18] to prove the existence of ground state solutions.

Next, the energy functional associated with (1.1) is given by

$$
J(u)=\frac{1}{2} \int_{\mathbb{R}^{N}}\left(1+2 u^{2}\right)|\nabla u|^{2}+\frac{1}{2} \int_{\mathbb{R}^{N}} u^{2}-\frac{1}{2} \int_{\mathbb{R}^{N}}\left(I_{\alpha} * G(u)\right) G(u) .
$$

To our aim, if we choose the variable $u=f(v)$ in $[7,12]$, then (1.1) reduces to

$$
-\Delta v+f(v) f^{\prime}(v)=\left(I_{\alpha} * G(f(v))\right) g(f(v)) f^{\prime}(v), \quad x \in \mathbb{R}^{N}
$$

where $f:[0,+\infty) \rightarrow \mathbb{R}$ is given by $f^{\prime}(t)=\frac{1}{\sqrt{1+2 f^{2}(t)}}$ on $[0,+\infty), f(0)=0$, and $f(-t)=f(t)$ on $(-\infty, 0]$. Based on the above facts, if $v$ is a weak solution of (1.3), then $u=f(v)$ is a weak solution of (1.1). The energy functional $J$ reduces to the following functional:

$$
\Phi(v)=\frac{1}{2} \int_{\mathbb{R}^{N}}\left(|\nabla v|^{2}+f^{2}(v)\right)-\frac{1}{2} \int_{\mathbb{R}^{N}}\left(I_{\alpha} * G(f(v))\right) G(f(v)) .
$$


Before stating our results, we need to define the set $\mathcal{Q}=\left\{v \in H^{1}\left(\mathbb{R}^{N}\right) \backslash\{0\}: \mathcal{P}(v)=0\right\}$, where $\mathcal{P}$ is given in Lemma 2.2. Now, we give our result in the following.

Theorem 1.1 Assume that $\left(g_{1}\right)-\left(g_{4}\right)$ are satisfied. Then problem (1.1) has a ground state solution $u=f(v)$ such that $\Phi(v)=\inf _{\mathcal{Q}} \Phi=\inf _{v \in \Theta} \max _{t>0} \Phi\left(v_{t}\right)>0$, where $v_{t}=v(x / t)$ and

$$
\Theta:=\left\{v \in H^{1}\left(\mathbb{R}^{N}\right): \int_{\mathbb{R}^{N}}\left[\frac{1}{2} f^{2}(v)-\frac{N+\alpha}{2}\left(I_{\alpha} * G(f(v))\right) G(f(v))\right]<0\right\} .
$$

\section{Notions}

- Let $H^{1}\left(\mathbb{R}^{N}\right)=\left\{u \in L^{2}\left(\mathbb{R}^{N}\right): \nabla u \in L^{2}\left(\mathbb{R}^{N}\right)\right\}$ with the norm $\|u\|=\left(\int_{\mathbb{R}^{N}}\left(|\nabla u|^{2}+u^{2}\right)\right)^{\frac{1}{2}}$.

- The embedding $H^{1}\left(\mathbb{R}^{N}\right) \hookrightarrow L^{s}\left(\mathbb{R}^{N}\right)$ is continuous for $s \in\left[2,2^{*}\right]$ and $H_{r}^{1}\left(\mathbb{R}^{N}\right) \hookrightarrow L^{s}\left(\mathbb{R}^{N}\right)$ is compact for $s \in\left(2,2^{*}\right)$.

- $H^{1}\left(\mathbb{R}^{N}\right) \hookrightarrow L^{\frac{2 N q}{N+\alpha}}\left(\mathbb{R}^{N}\right)$ if and only if $\frac{N+\alpha}{N} \leq q \leq \frac{N-2}{N+\alpha}$ (see [16]).

- $L^{p}\left(\mathbb{R}^{N}\right)$ denotes the usual Lebesgue space with norms $\|u\|_{p}=\left(\int_{\mathbb{R}^{N}}|u|^{p}\right)^{\frac{1}{p}}$, where $1 \leq p<\infty$.

- $\int_{\mathbb{R}^{N}}$ denotes $\int_{\mathbb{R}^{N}} \mathrm{~d} x$ and $C$ possibly denotes the different constants.

\section{Proof of Theorem 1.1}

In this section, we give the proof of Theorem 1.1. Next, let us recall some properties of the variables $f: \mathbb{R} \rightarrow \mathbb{R}$. These properties have been proved in $[7,12]$.

Lemma $2.1([7,12])$ The function $f(t)$ and its derivative satisfy the following properties:

(1) $f(t) / t \rightarrow 1$ as $t \rightarrow 0$;

(2) $f(t) \leq|t|$ for any $t \in \mathbb{R}$

(3) $f(t) \leq 2^{\frac{1}{4}} \sqrt{|t|}$ for all $t \in \mathbb{R}$;

(4) $f^{2}(t) / 2 \leq t f(t) f^{\prime}(t) \leq f^{2}(t)$ for all $t \in \mathbb{R}$;

(5) there exists a constant $C>0$ such that

$$
|f(t)| \geq \begin{cases}C|t|, & \text { if }|t| \leq 1 \\ C|t|^{\frac{1}{2}}, & \text { if }|t| \geq 1\end{cases}
$$

(6) $\left|f(t) f^{\prime}(t)\right| \leq \frac{1}{\sqrt{2}}$ for all $t \in \mathbb{R}$.

By the standard argument in $[16,19]$, we have the following Pohozaev type identity.

Lemma 2.2 If $v \in H^{1}\left(\mathbb{R}^{N}\right)$ is a critical point of (1.3), then $v$ satisfies

$$
\mathcal{P}(v):=\frac{N-2}{2} \int_{\mathbb{R}^{N}}|\nabla v|^{2}+\frac{N}{2} \int_{\mathbb{R}^{N}} f^{2}(v)-\frac{N+\alpha}{2} \int_{\mathbb{R}^{N}}\left(I_{\alpha} * G(f(v))\right) G(f(v))=0 .
$$

Motivated by [18], by a simple calculation, for any $t \in[0,1) \cup(1,+\infty)$, one has

$$
\begin{aligned}
& \beta(t):=\alpha+2-(N+\alpha) t^{N-2}+(N-2) t^{N+\alpha}>0 \text { and } \\
& \alpha-(N+\alpha) t^{N}-N\left(1-t^{N+\alpha}\right)>0 .
\end{aligned}
$$


Lemma 2.3 Assume that $\left(g_{1}\right)-\left(g_{4}\right)$ hold. Then, for all $v \in H^{1}\left(\mathbb{R}^{N}\right)$ and $t>0$,

$$
\Phi(v) \geq \Phi\left(v_{t}\right)+\frac{1-t^{N+\alpha}}{N+\alpha} \mathcal{Q}(v)+\frac{\beta(t)}{2(N+\alpha)}\|\nabla v\|_{2}^{2} .
$$

Proof From (1.4), we have

$$
\Phi\left(v_{t}\right)=\frac{t^{N-2}}{2} \int_{\mathbb{R}^{N}}|\nabla v|^{2}+\frac{t^{N}}{2} \int_{\mathbb{R}^{N}} f^{2}(v)-\frac{t^{N+\alpha}}{2} \int_{\mathbb{R}^{N}}\left(I_{\alpha} * G(f(v))\right) G(f(v)) .
$$

Thus, by (2.2), we have

$$
\begin{aligned}
& \Phi(v)-\Phi\left(v_{t}\right) \\
&=\frac{1-t^{N-2}}{2} \int_{\mathbb{R}^{N}}|\nabla v|^{2}+\frac{1-t^{N}}{2} \int_{\mathbb{R}^{N}} f^{2}(v)-\frac{1-t^{N+\alpha}}{2} \int_{\mathbb{R}^{N}}\left(I_{\alpha} * G(f(v))\right) G(f(v)) \\
&=\frac{1-t^{N+\alpha}}{N+\alpha} \mathcal{Q}(v)+\frac{\alpha+2-(N+\alpha) t^{N-2}+(N-2) t^{N+\alpha}}{2(N+\alpha)}\|\nabla v\|_{2}^{2} \\
& \quad+\frac{\alpha-(N+\alpha) t^{N}-N\left(1-t^{N+\alpha}\right)}{N+\alpha}\|f(v)\|_{2}^{2} \\
& \geq \frac{1-t^{N+\alpha}}{N+\alpha} \mathcal{Q}(v)+\frac{\beta(t)}{2(N+\alpha)}\|\nabla v\|_{2}^{2} .
\end{aligned}
$$

The proof is completed.

Corollary 2.4 Assume that $\left(g_{1}\right)-\left(g_{4}\right)$ hold. Then, for any $v \in \mathcal{Q}, \Phi(v)=\max _{t>0} \Phi\left(v_{t}\right)$.

Lemma 2.5 Assume that $\left(g_{1}\right)-\left(g_{4}\right)$ hold. Then, for any $\Theta \neq \emptyset$ and the set

$$
\left\{v \in H^{1}\left(\mathbb{R}^{N}\right) \backslash\{0\}: \mathcal{P}(v) \leq 0\right\} \subset \Theta .
$$

Proof By using $\left(g_{4}\right)$ and the method in [17] and [18], it follows that $\Theta \neq \emptyset$. Next, for any $v \in H^{1}\left(\mathbb{R}^{N}\right) \backslash\{0\}$, it follows from $\mathcal{P}(v) \leq 0$ that

$$
\frac{N}{2} \int_{\mathbb{R}^{N}} f^{2}(v)-\frac{N+\alpha}{2} \int_{\mathbb{R}^{N}}\left(I_{\alpha} * G(f(v))\right) G(f(v)) \leq-\frac{N-2}{2} \int_{\mathbb{R}^{N}}|\nabla v|^{2}<0,
$$

which shows that $v \in \Theta$. The proof is completed.

Lemma 2.6 Assume that $\left(g_{1}\right)-\left(g_{4}\right)$ hold. Then, for any $v \in \Theta$, there exists unique $t_{v}>0$ such that $v_{t_{v}} \in \mathcal{Q}$.

Proof Let $v \in \Theta$ be fixed. Set $\Gamma(t):=\Phi\left(v_{t}\right)$. Then it follows from $\Gamma^{\prime}(t)=0$ that

$$
\frac{N-2}{2} t^{N-3} \int_{\mathbb{R}^{N}}|\nabla v|^{2}+\frac{N t^{N-1}}{2} \int_{\mathbb{R}^{N}} f^{2}(v)-\frac{(N+\alpha) t^{N+\alpha-1}}{2} \int_{\mathbb{R}^{N}}\left(I_{\alpha} * G(f(v)) G(f(v))=0 .\right.
$$

Then

$$
\frac{N-2}{2} t^{N-2} \int_{\mathbb{R}^{N}}|\nabla v|^{2}+\frac{N t^{N}}{2} \int_{\mathbb{R}^{N}} f^{2}(v)-\frac{(N+\alpha) t^{N+\alpha}}{2} \int_{\mathbb{R}^{N}}\left(I_{\alpha} * G(f(v)) G(f(v))=0,\right.
$$


which implies that $\mathcal{P}\left(v_{t}\right)=0 \Leftrightarrow v_{t} \in \mathcal{Q}$. It is easy to check that $\lim _{t \rightarrow 0} \Gamma(t)=0, \Gamma(t)>0$ for $t>0$ enough small and $\Gamma(t)<0$ for $t$ large. Thus $\max _{t>0} \Gamma(t)$ is achieved at some $t_{v}>0$ such that $\Gamma^{\prime}\left(t_{v}\right)=0$ and $v_{t_{v}} \in \mathcal{Q}$.

Next, we will prove the uniqueness. For any given $v \in \Theta$, if there exist $t_{1}, t_{2}>0$ such that $v_{t_{1}}, v_{t_{2}} \in \mathcal{Q}$. Thus $\mathcal{P}\left(v_{t_{1}}\right)=\mathcal{P}\left(v_{t_{2}}\right)=0$. Therefore, we have

$$
\Phi\left(v_{t_{1}}\right) \geq \Phi\left(v_{t_{2}}\right)+\frac{t_{1}^{N}-t_{2}^{N}}{(N+\alpha) t_{1}^{N}} \mathcal{P}\left(v_{t_{1}}\right)+\frac{\beta\left(t_{2} / t_{1}\right)}{2(N+\alpha)}\left\|\nabla v_{t_{1}}\right\|_{2}^{2}=\Phi\left(v_{t_{2}}\right)+\frac{\beta\left(t_{2} / t_{1}\right)}{2(N+\alpha)}\left\|\nabla v_{t_{1}}\right\|_{2}^{2}
$$

and

$$
\Phi\left(v_{t_{2}}\right) \geq \Phi\left(v_{t_{1}}\right)+\frac{t_{2}^{N}-t_{1}^{N}}{(N+\alpha) t_{2}^{N}} \mathcal{P}\left(v_{t_{2}}\right)+\frac{\beta\left(t_{1} / t_{2}\right)}{2(N+\alpha)}\left\|\nabla v_{t_{2}}\right\|_{2}^{2}=\Phi\left(v_{t_{1}}\right)+\frac{\beta\left(t_{1} / t_{2}\right)}{2(N+\alpha)}\left\|\nabla v_{t_{2}}\right\|_{2}^{2},
$$

which shows that $t_{1}=t_{2}$. Thus $t_{v}>0$ is unique for $v \in \mathcal{Q}$. This completes the proof.

Lemma 2.7 Assume that $\left(g_{1}\right)-\left(g_{3}\right)$ hold, then $\mathcal{Q} \neq \emptyset$ and

$$
\inf _{\mathcal{M}} \Phi:=c=\inf _{v \in \Theta} \max _{t>0} \Phi\left(v_{t}\right)
$$

Proof This result is a consequence of Corollary 2.4, Lemma 2.5, and Lemma 2.6. The proof is completed.

By a standard argument in [19], we can get the following Brezis-Lieb lemma.

Lemma 2.8 Assume that $\left(g_{1}\right)-\left(g_{4}\right)$ hold. If $v_{n} \rightarrow v_{0}$ in $H^{1}\left(\mathbb{R}^{N}\right)$, then

$$
\Phi\left(v_{n}\right)=\Phi\left(v_{0}\right)+\Phi\left(v_{n}-v_{0}\right)+o_{n}(1)
$$

and

$$
\mathcal{P}\left(v_{n}\right)=\mathcal{P}\left(v_{0}\right)+\mathcal{P}\left(v_{n}-v_{0}\right)+o_{n}(1)
$$

Lemma 2.9 Assume that $\left(g_{1}\right)-\left(g_{4}\right)$ hold. Then

(i) there exists $\rho>0$ such that $\|\nabla v\|_{2} \geq \rho$ for any $v \in \mathcal{Q}$;

(ii) $c=\inf _{\mathcal{Q}} \Phi>0$.

Proof (i) By $\left(g_{3}\right)$, for any $\varepsilon>0$, there exists $C_{\varepsilon}^{1}>0$ such that

$$
|G(f(v))|^{\frac{2 N}{N+\alpha}} \leq \varepsilon|v|^{2}+C_{\varepsilon}^{1}|s|^{2^{*}} \text { and }|G(f(v))|^{\frac{2 N}{N+\alpha}} \leq \varepsilon|v|^{2}+\varepsilon|s|^{2^{*}}+C_{\varepsilon}^{1}|s|^{p},
$$

where $p \in\left(2,2^{*}\right)$. For any $v \in \mathcal{Q}$, we have that $\mathcal{P}(v)=0$. By the Sobolev embedding theorem, the Hardy-Littlewood-Sobolev inequality in [15], (2.3), and $\left(g_{1}\right)$, we get

$$
\begin{aligned}
& \frac{(N-2)}{2} \int_{\mathbb{R}^{N}}|\nabla v|^{2}+\frac{N}{2} \int_{\mathbb{R}^{N}} f^{2}(v) \\
& =\frac{N+\alpha}{2} \int_{\mathbb{R}^{N}}\left(I_{\alpha} * G(f(v))\right) G(f(v))
\end{aligned}
$$




$$
\leq C\left(\varepsilon \int_{\mathbb{R}^{N}}|f(v)|^{2}+C_{\varepsilon}^{1} \int_{\mathbb{R}^{N}}|v|^{2^{*}}\right) \leq C\left(\varepsilon \int_{\mathbb{R}^{N}}|f(v)|^{2}+C_{\varepsilon}^{1}\left(\int_{\mathbb{R}^{N}}|\nabla v|^{2}\right)^{2^{*} / 2}\right)
$$

which shows that there exists $\rho>0$ such that $\|\nabla v\|^{2} \geq \rho$ for any $v \in \mathcal{Q}$.

(ii) For any $v \in \mathcal{Q}$, from Lemma 2.2, we have

$$
\Phi(v)=\Phi(v)-\frac{1}{N+\alpha} \mathcal{P}(v) \geq \frac{\alpha+2}{2(N+\alpha)}\|\nabla v\|_{2}^{2} .
$$

This completes the proof.

Lemma 2.10 Assume that $\left(g_{1}\right)-\left(g_{3}\right)$ hold. Then $c$ is achieved.

Proof Let $\left\{v_{n}\right\} \subset \mathcal{Q}$ be a minimizer for $c$, that is, $\mathcal{P}\left(v_{n}\right)=0$ and $\Phi\left(v_{n}\right) \rightarrow c$ as $n \rightarrow \infty$. By (2.4), one has

$$
c+o_{n}(1)=\Phi\left(v_{n}\right)-\frac{1}{N+\alpha} \mathcal{P}\left(v_{n}\right)=\frac{\alpha+2}{2(N+\alpha)}\left\|\nabla v_{n}\right\|_{2}^{2}+\frac{N}{2(N+\alpha)} \int_{\mathbb{R}^{N}} f^{2}\left(v_{n}\right),
$$

which shows that $\int_{\mathbb{R}^{N}}\left|\nabla v_{n}\right|^{2}+\int_{\mathbb{R}^{N}} f^{2}\left(v_{n}\right)$ is bounded and thus $\left\{v_{n}\right\}$ is bounded in $D^{1,2}\left(\mathbb{R}^{N}\right)$. By the Sobolev inequality, Lemma 2.1-(5), it follows that

$$
\int_{\left|v_{n}\right| \leq 1} v_{n}^{2} \leq \int_{\mathbb{R}^{N}} f^{2}\left(v_{n}\right) \quad \text { and } \quad \int_{\left|v_{n}\right|>1} v_{n}^{2} \leq \int_{\left|v_{n}\right|>1} v_{n}^{2^{*}} \leq C\left(\int_{\mathbb{R}^{N}}\left|\nabla v_{n}\right|^{2}\right)^{2^{*} / 2}
$$

Therefore

$$
\int_{\mathbb{R}^{N}} v_{n}^{2}=\int_{\left|v_{n}\right| \leq 1} v_{n}^{2}+\int_{\left|v_{n}\right|>1} v_{n}^{2} \leq \int_{\mathbb{R}^{N}} f^{2}\left(v_{n}\right)+C\left(\int_{\mathbb{R}^{N}}\left|\nabla v_{n}\right|^{2}\right)^{2^{*} / 2} .
$$

From (2.5), we infer that there exists $C>0$ such that $\int_{\mathbb{R}^{N}} v_{n}^{2} \leq C$. Up to a subsequence, there exists $v_{0} \in H^{1}\left(\mathbb{R}^{N}\right)$ such that $v_{n} \rightarrow v_{0}$ in $H^{1}\left(\mathbb{R}^{N}\right), v_{n} \rightarrow v_{0}$ in $L_{\text {loc }}^{r}\left(\mathbb{R}^{N}\right)$ for $r \in\left[2,2^{*}\right)$ and $v_{n} \rightarrow v_{0}$ a.e. on $\mathbb{R}^{N}$.

Now, we claim that there exist $\delta>0$ and $\left\{y_{n}\right\} \subset \mathbb{R}^{N}$ such that $\int_{B_{1}\left(y_{n}\right)}\left|v_{n}\right|^{2}>\delta$. Assume by contradiction, by Lion's concentration compactness lemma in [19], that $v_{n} \rightarrow 0$ in $L^{r}\left(\mathbb{R}^{N}\right)$ for $2<r<2^{*}$. Moreover, by $\mathcal{P}\left(v_{n}\right)=0$, we know that

$$
\begin{aligned}
0 & \leftarrow \int_{\mathbb{R}^{N}}\left(I_{\alpha} * G\left(f\left(v_{n}\right)\right)\right) G\left(f\left(v_{n}\right)\right) \\
& =\frac{N-2}{N+\alpha} \int_{\mathbb{R}^{N}}\left|\nabla v_{n}\right|^{2}+\frac{N}{N+\alpha} \int_{\mathbb{R}^{N}} f^{2}\left(v_{n}\right) \geq \frac{N-2}{N+\alpha} \rho^{2}>0,
\end{aligned}
$$

as $n \rightarrow+\infty$. This is a contradiction. Thus there exist $\delta>0$ and $\left\{y_{n}\right\} \subset \mathbb{R}^{N}$ such that $\int_{B_{1}\left(y_{n}\right)}\left|v_{n}\right|^{2}>\delta$. Set $\bar{v}_{n}(x)=v_{n}\left(x+y_{n}\right)$. Then $\left\|\bar{v}_{n}\right\|=\left\|v_{n}\right\|$. Thus, up to a subsequence, there exists $\bar{v}_{0} \in H^{1}\left(\mathbb{R}^{N}\right) \backslash\{0\}$ such that $\bar{v}_{n} \rightarrow \bar{v}_{0}$ in $H^{1}\left(\mathbb{R}^{N}\right), \bar{v}_{n} \rightarrow \bar{v}_{0}$ in $L_{\text {loc }}^{r}\left(\mathbb{R}^{N}\right)$ for $r \in\left[2,2^{*}\right)$, and $\bar{v}_{n} \rightarrow \bar{v}_{0}$ a.e. on $\mathbb{R}^{N}$. By translation invariance, one has

$$
\Phi\left(\bar{v}_{n}\right) \rightarrow c, \quad \mathcal{P}\left(\bar{v}_{n}\right) \rightarrow 0, \quad \text { as } n \rightarrow+\infty
$$


and $\int_{B_{1}(0)}\left|\bar{v}_{n}\right|^{2}>\delta$. Set $\bar{w}_{n}:=\bar{v}_{n}-\bar{v}_{0}$. Thus Lemma 2.7 yields that

$$
c=\Phi\left(\bar{v}_{0}\right)+\Phi\left(\bar{w}_{n}\right)+o_{n}(1) \quad \text { and } \quad 0=\mathcal{P}\left(\bar{v}_{0}\right)+\mathcal{P}\left(\bar{w}_{n}\right)+o_{n}(1)
$$

If there exists a subsequence $\left\{\bar{w}_{n_{i}}\right\}$ of $\left\{\bar{w}_{n}\right\}$ such that $\bar{w}_{n_{i}}=0$, then up to a subsequence, we have

$$
\Phi\left(\bar{v}_{0}\right)=c, \quad \mathcal{P}\left(\bar{v}_{0}\right)=0 .
$$

Next, we assume that $\bar{w}_{n} \neq 0$. We claim that $\mathcal{P}\left(\bar{v}_{0}\right) \leq 0$. Otherwise, if $\mathcal{P}\left(\bar{v}_{0}\right)>0$, it follows from (2.7) that $\mathcal{P}\left(\bar{w}_{n}\right)<0$ for $n$ large. By virtue of Lemma 2.6, there exists $t_{n}>0$ such that $\left(\bar{w}_{n}\right)_{t_{n}} \in \mathcal{Q}$. By $(2.7)$ and Lemma 2.2 , we get

$$
\begin{aligned}
c & -\frac{N-2}{N+\alpha} \int_{\mathbb{R}^{N}}\left|\nabla \bar{v}_{0}\right|^{2}-\frac{N}{N+\alpha} \int_{\mathbb{R}^{N}} f^{2}\left(\bar{v}_{0}\right)+o_{n}(1) \\
& =\Phi\left(\bar{w}_{n}\right)-\frac{1}{N+\alpha} \mathcal{P}\left(\bar{w}_{n}\right) \geq \Phi\left(\left(\bar{w}_{n}\right)_{t_{n}}\right)-\frac{t_{n}^{N+\alpha}}{N+\alpha} \mathcal{P}\left(\bar{w}_{n}\right) \geq c-\frac{t_{n}^{N+\alpha}}{N+\alpha} \mathcal{P}\left(\bar{w}_{n}\right) \geq c,
\end{aligned}
$$

which is a contradiction due to $\int_{\mathbb{R}^{N}}\left|\nabla \bar{v}_{0}\right|^{2}>0$. Thus $\mathcal{P}\left(\bar{v}_{0}\right) \leq 0$. Since $\bar{v}_{0} \neq 0$, in view of Lemma 2.6, there exists $t_{0}>0$ such that $\left(\bar{v}_{0}\right)_{t_{0}} \in \mathcal{Q}$. By Lemma 2.3 and the weak semicontinuity of norm, we have

$$
\begin{aligned}
c & =\lim _{n \rightarrow \infty}\left[\Phi\left(\bar{v}_{n}\right)-\frac{1}{N+\alpha} \mathcal{P}\left(\bar{v}_{n}\right)\right] \\
& =\frac{N-2}{N+\alpha} \lim _{n \rightarrow \infty} \int_{\mathbb{R}^{N}}\left|\nabla \bar{v}_{n}\right|^{2}+\frac{N}{N+\alpha} \lim _{n \rightarrow \infty} \int_{\mathbb{R}^{N}} f^{2}\left(\bar{v}_{n}\right) \\
& \geq \frac{N-2}{N+\alpha} \int_{\mathbb{R}^{N}}\left|\nabla \bar{v}_{0}\right|^{2}+\frac{N}{N+\alpha} \int_{\mathbb{R}^{N}} f^{2}\left(\bar{v}_{0}\right) \\
& \geq \Phi\left(\bar{v}_{0}\right)-\frac{t_{0}^{N+\alpha}}{N+\alpha} \mathcal{P}\left(\bar{v}_{0}\right) \\
& \geq \Phi\left(\left(\bar{v}_{0}\right)_{t_{0}}\right)-\frac{t_{0}^{N+\alpha}}{N+\alpha} \mathcal{P}\left(\bar{v}_{0}\right) \\
& \geq c-\frac{t_{0}^{N+\alpha}}{N+\alpha} \mathcal{P}\left(\bar{v}_{0}\right) \geq c
\end{aligned}
$$

which implies that (2.8) holds. The proof is completed.

By a standard argument in $[14,18,19]$, we can get the following lemma.

Lemma 2.11 Assume that $\left(g_{1}\right)-\left(g_{4}\right)$ hold. If $\tilde{v} \in \mathcal{Q}$ and $\Phi(\tilde{v})=c$, then $\tilde{v}$ is a critical point of $\Phi$.

Proof of Theorem 1.1 By Lemma 2.7, Lemma 2.10, and Lemma 2.11, there exists $v_{0} \in \mathcal{Q}$ such that

$$
\Phi\left(v_{0}\right)=c=\inf _{v \in \Theta} \max _{t>0} \Phi\left(v_{t}\right), \quad \Phi^{\prime}\left(v_{0}\right)=0 .
$$

This completes the proof. 


\section{Acknowledgements}

The authors would like to express sincere thanks to the anonymous referees for their careful reading of the manuscript and valuable comments and suggestions.

\section{Funding}

This work was supported by the NSFC (No. 11501232), NSF of Hunan Province (No. 2017JJ2213), Research Foundation of Education Bureau of Hunan Province, China(Nos. 18A452, 19B450, 19C1465), Huaihua University Scientific Research Project (No.HHUY2019-3), and Huaihua University Double First-Class Initiative Applied Characteristic Discipline of Control Science and Engineering.

\section{Availability of data and materials}

Not applicable.

\section{Competing interests}

The authors declare that they have no competing interests.

\section{Authors' contributions}

The authors declare that this study was independently finished. All authors read and approved the final manuscript.

\section{Authors' information}

Not applicable.

\section{Publisher's Note}

Springer Nature remains neutral with regard to jurisdictional claims in published maps and institutional affiliations.

Received: 15 April 2020 Accepted: 25 June 2020 Published online: 06 July 2020

\section{References}

1. Bass, F.G., Nasanov, N.N.: Nonlinear electromagnetic-spin waves. Phys. Rep. 189, 165-223 (1990)

2. Berestycki, H., Lions, P.: Nonlinear scalar field equations, I. Existence of a ground state. Arch. Ration. Mech. Anal. 82, 313-345 (1983)

3. Chen, J.H., Cheng, B.T., Huang, X.J.: Ground state solutions for a class of quasilinear Schrödinger equations with Choquard type nonlinearity. Appl. Math. Lett. 102, 106141 (2020)

4. Chen, S., Wu, X.: Existence of positive solutions for a class of quasilinear Schrödinger equations of Choquard type. J. Math. Anal. Appl. 475, 1754-1777 (2019)

5. Chen, S.T., Tang, X.H.: Ground state solutions for generalized quasilinear Schrödinger equations with variable potentials and Berestycki-Lions nonlinearities. J. Math. Phys. 59(8), 081508 (2018)

6. Chen, S.T., Tang, X.H.: Ground state solutions for general Choquard equations with a variable potential and a local nonlinearity. Rev. R. Acad. Cienc. Exactas Fís. Nat., Ser. A Mat. 114(14), 1-23 (2020)

7. Colin, M., Jeanjean, L.: Solutions for a quasilinear Schrödinger equation: a dual approach. Nonlinear Anal. 56, 213-226 (2004)

8. Fang, X., Szulkin, A.: Multiple solutions for a quasilinear Schrödinger equation. J. Differ. Equ. 254, 2015-2032 (2013)

9. Goldman, M.V.: Strong turbulence of plasma waves. Rev. Mod. Phys. 56, 709-735 (1984)

10. Jeanjean, L.: On the existence of bounded Palais-Smale sequences and application to a Landesman-Lazer type problem set on $\mathbb{R}^{N}$. Proc. R. Soc. Edinb. A 129, 787-809 (1999)

11. Kurihara, S.: Large-amplitude quasi-solitons in superfluid films. J. Phys. Soc. Jpn. 50, 3262-3267 (1981)

12. Liu, J.Q., Wang, Y., Wang, Z.Q.: Solutions for quasilinear Schrödinger equations, II. J. Differ. Equ. 187, 473-793 (2003)

13. Liu, X., Liu, J., Wang, Z.Q.: Quasilinear elliptic equations via perturbation method. Proc. Am. Math. Soc. 141, 253-263 (2013)

14. Luo, H.X.: Ground state solutions of Pohozaev type and Nehari type for a class of nonlinear Choquard equations. J. Math. Anal. Appl. 467, 842-862 (2018)

15. Makhankov, V.G., Fedyanin, V.K.: Nonlinear effects in quasi-one-dimensional models and condensed matter theory. Phys. Rep. 104, 1-86 (1984)

16. Moroz, V., Van Schaftingen, J.: Groundstates of nonlinear Choquard equations: existence, qualitative properties and decay asymptotics. J. Funct. Anal. 265, 153-184 (2013)

17. Moroz, V., Van Schaftingen, J.: Existence of groundstate for a class of nonlinear Choquard equations. Trans. Am. Math. Soc. 367, 6557-6579 (2015)

18. Tang, X.H., Chen, S.T.: Singularly perturbed Choquard equations with nonlinearity satisfying Berestycki-Lions assumptions. Adv. Nonlinear Anal. 9, 413-437 (2020)

19. Willem, M.: Minimax Theorems. Birkhäuser, Berlin (1996)

20. Wu, X.: Multiple solutions for quasilinear Schrödinger equations with a parameter. J. Differ. Equ. 256, 2619-2632 (2014)

21. Yang, X., Zhang, W., Zhao, F.: Existence and multiplicity of solutions for a quasilinear Choquard equation via perturbation method. J. Math. Phys. 59, 081503 (2018) 